\title{
Modelling gradient perception and polarization in chemotaxing Dictyostelium cells Jawahar Krishnan*
}

Address: Imperial College London, London, UK

Email: Jawahar Krishnan* - j.krishnan@imperial.ac.uk

* Corresponding author

from BioSysBio 2007: Systems Biology, Bioinformatics and Synthetic Biology Manchester, UK. II-13 January 2007

Published: 8 May 2007

BMC Systems Biology 2007, I(SuppI I):P7 doi:I0.I I86/I752-0509-I-SI-P7

This abstract is available from: http://www.biomedcentral.com/I752-0509/I?issue=SI

(c) 2007 Krishnan; licensee BioMed Central Ltd.

Chemotaxis, the directed migration of cells/organisms in response to external chemical gradients, is of great importance in various biological settings such as wound healing, tumour metastasis and development.

This talk deals with modelling efforts aimed at understanding two key sub-processes of eukaryotic chemotaxis: gradient perception, the process by which information about an external concentration field is converted into an internal signal which guides cell motion, and polarization, the persistent localization of various signalling molecules to opposite ends of the cell ("front" and "back"), along with any attendant morphological change.

We first briefly examine models for gradient perception in Dictyostelium. This involves the regulation of phosphoinositide lipids such as $\operatorname{PI}(3,4,5) \mathrm{P} 3 / \mathrm{PI}(3,4) \mathrm{P} 2$, by the receptor, via the enzymes PI3K and PTEN, in a manner consistent with adaptation to homogeneous stimuli. We then discuss a modelling framework developed to link gradient perception with cell polarization. While cell polarization occurs as a result of exposure to an external chemical gradient, there are intrinsic mechanisms which can also lead to cell polarization, which do not require an externally imposed chemical gradient.

Working within our modelling framework, we examine the interaction between external gradient-induced and intrinsic polarization. Model analysis leads to various testable predictions regarding their interaction and is a first step towards elucidating the complexities of the polarization process in this system. This is work done in collaboration with Pablo Iglesias and Peter Devreotes, Johns Hopkins University. 